\title{
Laparoscopic partial nephrectomy in the treatment of renal tumors in Thanh Hoa General Hospital, Vietnam
}

\author{
T.T. Tung ${ }^{1}$, H. Long ${ }^{2}$, L.Q. Hai ${ }^{1}$ and N.V. Thanh ${ }^{3}$ \\ ${ }^{1}$ Department of Urology of Thanh Hoa general hospital \\ ${ }^{2}$ Department of Emergency abdominal surgery of Viet Duc University \\ Hospital, Vietnam \\ ${ }^{3}$ Department of General surgery of Thanh Hoa city general hospital \\ Corresponding author: H. Long \\ E-mail: hoanglong70@gmail.com
}

Genet. Mol. Res. 20 (1): gmr18747

Received December 30, 2019

Accepted April 20, 2020

Final Revision January 25, 2021

Published March 30, 2021

DOI http://dx.doi.org/10.4238/gmr18747

\begin{abstract}
We evaluated the initial results of laparoscopic partial nephrectomy (LPN) in the treatment of renal tumors in Thanh Hoa General Hospital. During examination, 10 patients (6 females, 4 males) were diagnosed with renal tumors on CT scans. Later they underwent LPN at Thanh Hoa General Hospital from November 2016 to March 2018. The mean age was 54.5 years. Average tumor size was $3.5 \mathrm{~cm}$. There were seven patients in the pTla stage and 3 in the pT1b stage. The R.E.N.A.L. nephrometry scoring: two patients in $4 \mathrm{a}$; two patients in $4 \mathrm{p}$; three patients in $5 \mathrm{a}$; two patients in $6 \mathrm{p}$; and one patient in $8 \mathrm{x}$. Average operative time was 125.6 minutes. Average warm-ischemic time was 29.4 minutes. Blood loss average was $85.2 \mathrm{~mL}$. Hemorrhagic complication in one patient. Convert to laparoscopic radical nephrectomy in one patient. Patients were followed for one to three months without complications, metastases, or local recurrence.
\end{abstract}

Key words: Renal function; Partial nephrectomy; Radial nephrectomy; Renal tumor; Laparoscopic surgery 


\section{INTRODUCTION}

Laparoscopy in humans was first performed more than 100 years ago by Swedish surgeon Hans-Christian Jacobaeus; he named this procedure laparothorakoskopy (Guillonneau et al., 2009) The first renal surgery using this technique was performed in 1990 in the USA.

Nowadays, thanks to the widespread use of imaging tools, such as ultrasound and CT scans, the rate of early detection or accidental detection of small- size kidney tumors is on the rise. According to Rendon et al. (2014), the accidental detection rate of kidney tumors increased by about $2.3 \%$ per year. Partial nephrectomy $(\mathrm{PN})$ is a standard treatment option for kidney tumors $\leq 7 \mathrm{~cm}$ (stage pT1) with the same cancerous results as radical nephrectomy $(\mathrm{RN})$. The advantage of partial renal resection is that it saves and preserves the functional kidney units, so that in the long term less kidney replacement treatments will be needed than with radical kidney resection (Guillonneau et al., 2009; Dominguez-Escrig et al., 2011).

Along with the trend of developing less invasive surgery, LPN has many advantages: reducing hospital time; increase resilience of the patient; Low blood loss and high aesthetic, is now an appropriate alternative to open partial nephrectomy (OPN) in the treatment of kidney tumors. However, due to the technical and equipment challenges, this type of surgery still needs to be performed at large medical facilities because surgeons have experience in laparoscopic surgery. According to statistics from many data sources in 2007, the 5-year additional survival rate of cancer-free after endoscopic resection surgery was 86\% (Wolf, 2010; Dominguez-Escrig et al., 2011). It is generally believed that laparoscopic radical and partial nephrectomy can be performed through both transperitoneal and retroperitoneal approaches, and each approach has its own advantages and limitations.

In Vietnam, major surgical centers such as Viet Duc Hospital, Hue Central Hospital, Ho Chi Minh City Medical University Hospital, Cho Ray Hospital, and Binh Dan Hospital have performed semi-renal endoscopic surgery. in the treatment of kidney tumors relatively early. For provincial hospitals, this is still a challenge, and little is being done. Thanh Hoa General Hospital has used this type of surgery since the end of 2016. We conducted this study to evaluate the initial results of laparoscopic surgery in the treatment of kidney tumors at the provincial general hospital.

\section{MATERIAL AND METHODS}

\section{Subjects of research}

We included 10 patients (6 females, 4 males) diagnosed with kidney tumor on CT scan image and semi-laparoscopic surgery at Thanh Hoa Provincial General Hospital from November 2016 to March 2018.

All the participants agreed to take part in this experiment and agreed that the results of the study could be shown in a research paper. All manipulations below were in according to the moral standards of the institutional committee on human experimentation and with the Helsinki Declaration of 1975, as revised in 2000.

Basis for choosing to appoint laparoscopic resection surgery:Kidney tumor at pT1 stage (according to AJCC's stage of kidney cancer classification in 2010 (Rendon, 2014) has: pT1a is kidney tumor with size $\leq 4 \mathrm{~cm}$; pT1b is tumor with size $\leq 7 \mathrm{~cm}$; pT2 is block 
tumors that are $>7 \mathrm{~cm}$ ) or smaller and the patient has no contraindications to laparoscopic surgery.Calculating tumor complexity score according to the RENAL system of Kutikov and Uzzo in 2009 (this system is based on five criteria: tumor size; tumor convexity on the kidney surface; distance from tumor to pyelonephritis system; the tumor is located on the front or the back; the tumor location is related to the kidney pole. each criterion has from 1 to 3 points) (Kutikov and Uzzo, 2009) is low and moderate.

\section{Research methods}

Procedures were descriptive data research. The patients were diagnosed and surgeruy performed according to a procedure, using Karl Storz's Full HD laparoscope by the same surgeon, using a Hamonic scalpel Gen 11 ultrasound scalpel from Ethicon EndoSurgery, as well as Hem-o-lock clips of size 5 and $10 \mathrm{~mm}$.

Patient preparation: All patients underwent endotracheal anesthesia, gastric catheterization, urethral catheterization and intestinal cleansing prior to surgery.

Surgical procedure: All patients underwent laparoscopic endoscopic surgery to cut the kidneys in the opposite position to 900 with padded waist area.

Place the first trocar by the open method, create a cavity with a $600 \mathrm{ml}$ air pump glove, the rest of the trocar is placed under the observation of the lens, the position of the trocar at the front, middle and back axillary lines. Pump $\mathrm{CO}_{2}$ gas with pressure $\leq 12 \mathrm{mmHg}$, initial pump speed $2-31 / \mathrm{min}$.

For the right kidney surgery: the surgery reveals the kidney artery, reveals the kidney with tumor (it is best to completely show the kidney), marking the boundary to cut around the tumor with an electric knife. Monopolar (about $2-3 \mathrm{~mm}$ from the edge of the $\mathrm{u}$ ) Use a rubber strap to slip through the tight kidney artery and clamp with Hem-o-lock. Once the kidneys have softened, use scissors to cut tumor at the margin of the benign kidney (enucleation). Sutures of pyelonephritis with vicryl 3-0 or 2-0 only. Place the bolster with a rolled surgical plate. The kidney is restored with 2-0 or 1-0 vicryl stitches that use Hem-olocks to secure the thread head. Release the rubber strap on the renal artery, check the sectional hemostasis and additional hemostasis. Place drainage near the kidneys, close the 2-layer trocar.

For left side kidney surgery: same.

* Care and indicators to consider: Patients were monitored for pulse, arterial blood pressure, breathing, temperature and drainage postoperative status.

For antibiotics, we used the $\beta$ lactamine group in combination with the quinolon group. Non-steroidal medicines and morphine were used for pain. We recorded duration of surgery, duration of anemia, control of blood vessels, technique of renal rehabilitation, amount of blood lost, number of trocars, time of draining duct, time of hospitalization, urine leakage, and complications. We evaluated that patients at one and three months (clinical, ultrasound).

\section{RESULTS}

\section{Some pathological characteristics}


Ten patients successfully underwent LPN and completed a one year follow-up according to our protocol. Each patient was found to have a kidney tumor by accident, two patients had vague pain in the lumbar area, none had hematoma symptoms or a palpable lumbar tumor. Patients were screened for kidney tumors by ultrasound. We determined the stage of disease and the complexity of the tumor (nephrometry points) before surgery by CT scan.

\section{Table 1. Characteristics of the kidney tumor patients.}

\begin{tabular}{ll}
\hline Variable & Mean (range) or $\mathbf{n}$ or \% \\
\hline Age (years) & $54.5(38-67)$ \\
Sex (male/female) & $04 /$ jun \\
Located side (right/left) & $06 / \mathrm{abr}$ \\
Size of tumor (cm) & $3.5(3-5)$ \\
Location of tumor & \\
Upper polar & 3 \\
Middle part & 3 \\
Lower polar & 4 \\
Nephrometry score * & \\
4a & 2 \\
4p & 2 \\
5a & 3 \\
6p & 2 \\
8x & 1 \\
Staging** & \\
pT1a & 7 \\
pT1b & 3 \\
p pT2 & 3 \\
Histology & 0 \\
Clear cell & \\
Papillary & 5 \\
Chromophobe & 2 \\
Angiomyolipoma & 1 \\
\hline * Using nephrometry score of R.E.N.A.L. system (Nephrometric ball system & 2. R.N.A.L. (R.E.N.A.L. scale) allows one to assess the \\
complexity of performing a kidney resection and predict possible complications after performing organ-preserving surgery.
\end{tabular}

The system was developed using CT images, although MRI can also be used. Contrast enhancement is recommended. If contrast is contraindicated, unenhanced MRI can be used to determine nephrometry score.

" $\mathrm{R}$ " is the maximum diameter of the mass. Lesions $\leq 4 \mathrm{~cm}$ are assigned 1 point, $>4$, but $<7 \mathrm{~cm}$ are assigned 2 points, $\geq 7 \mathrm{~cm}-3$ points;

"E" - exophytic or endophytic location of the tumor. Lesions that are predominantly endophytic present a greater surgical problem than those that are exophytic. Lesions, more than $50 \%$ of the volume of which are located outside the renal cortex, are assigned 1 point, less than $50 \%-2$ points, and completely endophytic - 3 points;

"N" is the proximity to the collecting system, measured in millimeters. Best defined on excretory images. As with the "R" descriptor, the scale is divided between 4 and 7 using millimeters rather than centimeters. $7 \mathrm{~mm}$ or more from the collecting system or renal sinus - 1 point, $>4$ but $<7 \mathrm{~mm}-2$ points, $4 \mathrm{~mm}$ or less -3 points.

Descriptor "A" is determined from axial images, indicates anterior or posterior location of the tumor and is not scored. If the tumor lies mainly on the ventral surface of the kidney, the designation "a" is assigned. Tumors located on the dorsal surface of the kidney 
are designated "p". Tumors that do not fall into either of these categories (purely lateral or central apical lesion) are designated "x".

"L" - the position of the tumor relative to the polar lines. The superior and inferior polar lines are defined by the vascular pedicle of the kidney and can be identified on both axial and coronal images. Tumors that are completely above or below the polar lines are assigned a point of 1; if the lesion crosses the polar line, 2 points are assigned; and if $>50 \%$ of the volume of the mass crosses the polar line or the mass is completely between the polar lines, 3 points are assigned. Lesions that are adjacent to the renal vein or artery are given an " $\mathrm{h}$ " suffix to indicate their location at the renal hilum. This designation does not affect the score scale.

The sum of the points determines the complexity of the tumor:

low complexity (nephrometry score $=4-6$ );

moderate complexity (nephrometry score $=7-9$ );

high complexity (nephrometry score $=10-12$ );

** Staging follow AJCC 2010 (stage of kidney cancer classification in 2010 (Rendon, 2014) has: pT1a is kidney tumor with size $\leq 4 \mathrm{~cm}$; pT1b is tumor with size $\leq 7 \mathrm{~cm}$; pT2 is block tumors that are $>7 \mathrm{~cm}$ ).

Table 2. Surgical characteristics for the 10 kidney tumor patients.

\begin{tabular}{ll}
\hline Variable & Mean (range) or n \\
\hline Amount of trocars & 7 \\
3 & 3 \\
4 & $125.6(90-185)$ \\
Operative time (minutes) & $29.4(20-43)$ \\
Warm-ischaemic time * (minutes) & 10 \\
Main renal artery clamping & 0 \\
Hilar clamping en block & $85.2(50-250)$ \\
Blood loss (ml) & $5(4-6)$ \\
Time to drain (days) & $6(5-7)$ \\
Hospital stay (days) & 1 \\
Intraoperative complications ** & 0 \\
Postoperative complications & \\
Examine postoperatively *** & 10 patients \\
1 month & 6 patients \\
3 months &
\end{tabular}

In this series of patients, we observed that RFD depended on tumor size, warm ischemic time, duration of the surgery. The prognosis of renal tumor mainly depends on histology. As abdominal imaging is getting more and more popular the amount of small renal tumors has increased in recent decades.

Operative time is highly connected to other points, such as patient's weight etc.; these may require a longer surgery time.

Surgeons clamp renal artery to interrupt the blood flow to kidney during surgery, which helps the surgeon to work on the open area with less bleeding. There are two opinions about this action. Some authors say that blood flow interruption can be a reason of 
an ischemic damage, while others think that kidney can support it. Anyway, modern studies show its harmful effect on the kidney.

The greatest achievement of this method is that there were not postoperative complications in patients. Blood loss depends on some factors, such as tumor size, tumor complexity. These factors also effect operative time.

It is also important to emphasize the direct relationship between Warm - ischemic time (WIT) and tumor size. In our study it was appropriately 30 minutes.

For larger tumors, this time may be up to 50 minutes. Current studies aimed to differ the impact of parenchymal loss from the WIT effect on the operated kidney.

Tumor size and renal function are vital determinants of suitability for PN. The age, sex of patients, tumor location and other factors remain to stay important in postoperative outcomes. We believe that with the integration of new methods post-operative hospital stays would decrease.

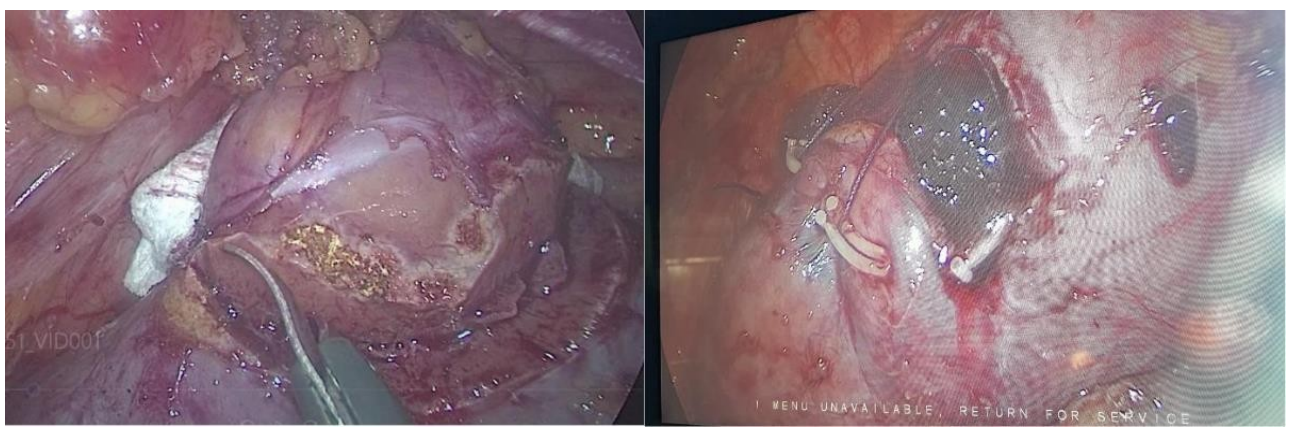

Figure 1. Kidney tumor is resected by cold scissors (A) and the kidney is sutured to recover (B).

\section{DISCUSSION}

PN in the treatment of kidney tumors has been carried out over the past two decades, with the same cancerous treatment results as radical nephrectomy, especially in the long term. The kidney will save and preserve the functional kidney unit. LPN is a minimally invasive procedure and may yield results that are not different from that of OPN. Therefore, LN is gradually being accepted and preferred for treatment of benign kidney tumors and pT1 or less stage kidney cancer (Guillonneau et al., 2009; Rendon et al., 2014).

Laparoscopic surgery approaches to the kidney during LPN presently include transperitoneal, retroperitoneal, hand-assisted, robotic, laparoendoscopic single-site surgery (LESS), and natural orifice transluminal endoscopic surgery (NOTES). With each approach, a pneumoperitoneum is created to increase the intra-abdominal working space.

Numerous studies have found that patients undergoing LPN had decreased rate of surgical complications, length of stay, need for blood transfusion etc. With recognition of this fact this method is getting more and more popular.

Laparoscopic renal surgery is far from being perfected. Physician's goals for the future are to improve outcomes, decrease length of recovery while trying to cost effective and as lest invasive as possible. 
Robotic technologies are being developed, which can assist in completing different operations. It includes image guided robots that can help to introduce instrument or needles into the kidney safely to avoid organ injury. Also in robot assisted partial nephrectomy (RAPN) tumor complexity does not affect operative time, while for LPN it does.

Under the guidance of the Canadian Cancer Society, for kidney tumors in the pT1a stage (tumor size $\leq 4 \mathrm{~cm}$ ), it is preferable to choose treatment with: partial renal resection; radiofrequency ablation (RFA) or cryoablation As for kidney tumors in the pT1b stage (tumor size $>4 \mathrm{~cm}$ and $\leq 7 \mathrm{~cm}$ ), you should only choose to have PN or RN. The choice of laparoscopic surgery or open surgery to remove the tumor or kidney depends on the ability of the surgeon and the facilities of the treatment facility (Dominguez-Escrig et al., 2011).

Recently, many authors have added to the tumor complexity scoring system (usually calculated by the RENAL system of Kutikov and Uzzo in 2009) to predict the difficulty of the PN process, especially is to PN by laparoscopic surgery, this system is based on 5 criteria to calculate points, each of the criteria has from 1 to 3 points, of which 2 criteria are the most noticeable, that is: size tumor size and the closest distance from the tumor to the renal pelvis system (nearness to the collecting system). For PN by laparoscopic surgery, patients with low-grade kidney tumors (4-6 points) and average (7-9 points) should be selected (Kutikov and Uzzo, 2009; Rendon et al., 2014).

The patients in our study group were assigned a PN with an average tumor size of $3.5 \mathrm{~cm}$, in which the proportion of tumors was accidentally discovered by ultrasonic examination is relatively high ( $8 / 10$ patients), the tumor complexity score calculated by the RENAL system from 4a to $8 x$, successful surgery results in 9/10 patients. About 1 in 10 patients has to change the method to radical nephrectomy by laparoscopic surgery, this is the case of a kidney tumor with a size of $5 \mathrm{~cm}$, located at the position crossing the polar lines and lying deep into renal parenchyma (endophytic). According to Kapoor, it is recommended that for new surgeons to perform LPN who should be selected patients with renal tumor in pT1a stage, tumors that are protruding out of the kidneys (exophytic) and not near the renal hilar (nonhilar) are needed, he also said that when the surgeon passes the first 10-20 cases, he should extend the appointment of LPN for larger and more complicated kidney tumors (Kapoor, 2009). According to Nadu et al. (2007), 140 cases of LPN showed that: for the first 30 cases, although only selected tumors with an average size of $\mathrm{cm}$ but the rate of transferring surgery method is up to $10 \%$; While with the next 110 cases, with an average tumor size of $3.9 \mathrm{~cm}$, the rate of transferring surgery method was only $2.7 \%$.

\section{The surgical process Access and trocars}

Choosing the way to enter the retroperitoneal or abdominal cavity to perform LPN is very important. According to the instructions in Laparoscopic Techniques in UroOncology, it is recommended to use the abdominal route for cases of anterior, anteriorlateral side tumors or large kidney tumors that require at least $30 \%$ of the kidneys. If the tumor is on the back or the posterior side, the retroperitoneal route should be used (Guillonneau et al., 2009). Recently, many authors have considered the choice of the route of entry for LPN, which often follows the habits of the surgeon and the views of the treatment facility. In this study, we performed all cases of LPN through the retroperitoneal line, which has the advantage of direct access to the renal artery to control. The cavity must 
be made to the maximum extent to facilitate the surgery process. The patients in our study had five cases of anterior tumor, four cases of tumor on the back and one case of tumor in the medial (lateral) position; all surgeries were performed by retroperitoneal LPN with 3-4 working trocars.

\section{Controlling kidney blood vessels and the time of hot anemia}

In LPN, the control of the blood vessels of the kidney will make the cutting process as well as the recovery suture of the kidneys less blood loss, the surgical field and the tumor area are clearer, but it is this control that makes the kidneys suffer from anemia during the procedure. The maximum time the kidney has a warm-ischaemic time (WIT) depends on the author, but it can be up to 30 minutes, which will limit the time it takes for the kidneys to be cut and restored, especially for large and complex kidney tumors (Guillonneau et al., 2009; Shikanov et al., 2010). Some authors believe that for kidney tumors that require a lot of time for cutting and recovery, they should lower the temperature of the kidneys before controlling blood vessels, at which time the kidneys will be cold anemia with the time of cold anemia (cold ischemic time - CIT) may last longer (Wolf, 2010). Recently, there have been authors reporting successful cases of super-selective control of tumor-specific arteries during renal laparoscopic surgery (Dominguez-Escrig et al., 2011). According to Wolf (2010) the viewpoint in LPN is: for kidney tumors with deep penetration into renal parenchyma (tumor depth) $>5 \mathrm{~mm}$, it is recommended to perform clamping pedicle of kidney; When $\leq 5 \mathrm{~mm}$, there is no need to clamp pedicle of kidney.

In our research patients, all cases were controlled renal pedicle by rubber band but still ensure good hemostasis required for surgery, there is no case of using lowering Kidney temperature or super-selective control of tumor arteries. The average duration of hot anemia of the patients in the study group was 29.4 minutes, in the case of hot anemia was 43 minutes, the tumor was large, deep in the kidney parenchyma, when the stage of kidney recovery was completed, drop control of kidney arteries to see a lot of bleeding, although the stitch has been supplemented but unable to control the bleeding, it is necessary to transfer to laparoscopic radical nephrectomy. The results of our hot anemia time were not significantly different from the other research of Tran Ngoc Sinh et al. (2010) - 10-20 minutes; Brown (2007) - 30 minutes (15 - 55); Nadu et al. (2007) - 30 minutes (18 - 49).

\section{Tumor removal and recovery of renal parenchyma}

Before the tumor is removed during LPN, the peripolar fat should be liberated from the kidney surface (taking care not to lose the renal cortex), but the adipose layer adjoins and adheres to the mass should be left with the purpose of facilitating the removal of the tumor and also to help the process of staging the disease on histopathology later on. The tumor was cut in a enucleation to the healthy kidney (note that the margin area must be negative), in order to do this well, many authors recommend using non disposable laparoscopic cold scissors, the process of attention is always controlled and controlled. These points break into the calyxes system or renal vessels to correct treatment during recovery. Most authors perform a double-layer suture using a bolster with rolled-up surgical pads, but for tumors that are small in size and less invasive into kidney parenchyma, it may not be necessary to use a bolster or even not renal parenchyma repair suture (Wolf, 2010). 
In this study, we stitched 2 layers using bolster in all cases with a success rate of 9/10 patients, 1 in 10 patients bleed immediately after release controlled arteries, in this case, due to the large tumor and deep invasion of the kidney sinus (RENAL score $=8 \mathrm{x}$ ), when cutting, it has violated large artery branches, causing failure of the process of recovery and hemostasis.

\section{Some surgical results}

The result of our average operation time is 125.6 minutes. The average blood loss was $85.2 \mathrm{ml}$. The draining period and the average hospitalization time were 5 and 6 days, respectively. Histological results: kidney cancer accounts for $8 / 10$ cases, benign kidney $2 / 10$ cases. Follow-up after 1 month in 10 patients, after 3 months in 6 patients, all patients did not have urine leakage, hernia or infection of trocar, especially no patients showed any signs of metastasize far or locally.

There was one case of bleeding during surgery that required surgical procedure to laparoscopic radical nephrectomy, this case took about $250 \mathrm{ml}$ of blood and had the longest surgery time (185 minutes). Although the outcome of the surgery is still goal and safe for the patient (not a blood transfusion), if we have better assessments to change the procedure right from the beginning or when cutting the tumor, the patient does not lost a lot of blood and did not last long. Therefore, it is very important to complete bilan before surgery to evaluate and plan for a LPN, Wolf (2010) recommends that surgeons:

Before surgery, procedure should be based on image diagnosis to evaluate: the deep invasion of the tumor into renal parenchyma; the closest distance from the tumor to the calyxes system; and the location of the tumor.

During surgery, an endoscopic ultrasound should be used to assess the association of the tumor with the calyxes system, sinuses and blood vessels.

The surgeon must know how to use additional means and techniques to stop bleeding: biological glue; techniques to control blood vessels; and technique of placing stitches or bolster.

LPN is an ideal option for tumors in initial stages, because it reduces the possible risk of glomerular filtration decrease. In the treatment of renal tumors, it is a surgical procedure that can be performed at provincial hospitals, with initial options for renal tumors of size $\leq 4 \mathrm{~cm}$. However, in order to perform well using this technique, the facility must have adequate equipment, laparoscopic experienced surgeons and intensive training. Nevertheless, this method seems to lead to more post-operative complications, needing longer warm ischemic time with corresponding renal function deterioration (RFD). Nonetheless, various studies have shown less risk of chronic kidney disease and cardiovascular events after LPN, with a noteworthy improvement in the overall survival rate. One of the main aims of this study was to decrease the loss of renal function associated with LPN. Quantity and quality of the saved renal parenchyma are two fundamental criteria that evaluate post-operative renal function.

\section{CONCLUSIONS}

LPN for the treatment of kidney tumors is a type of surgery that can be performed at provincial hospitals, with an appropriate initial selection of $\leq 4 \mathrm{~cm}$ kidney tumors. However, 
to perform this technique well, the treatment facilities need to have adequate equipment, surgeons with experience in laparoscopic surgery and intensive training.

With the help of technology, renal surgery is now significantly less invasive, and operative outcomes are more positive. As modern technology develops, alternatives will continue to be sought by urologists with the hope of improvement of patient outcomes and satisfaction.

\section{ACKNOWLEDGMENTS}

The authors would like to thank the Department of Urology, Thanh Hoa Provincial General Hospital for the support during the research.

\section{CONFLICTS OF INTEREST}

The authors declare no conflict of interest.

\section{REFERENCES}

Benway BM, Bhayani SB, Rogers CG, Dulabon LM, et al. (2009). Robot assisted partial nephrectomy versus laparoscopic partial nephrectomy for renal tumors: a multi-institutional analysis of perioperative outcomes. $J$. Urol. 182(3): 866-873.

Bolton EM and Lynch TH (2018). Impact of warm ischaemia time during partial nephrectomy on renal function-is it really a matter of time? BJU Int. 121(1): 3-4.

Brown GA (2007). Laparoscopic partial nephrectomy: Experience in 60 cases. J. Endourol. 21: 71-74.

Cwach K and Kavoussi L (2016). Past, present, and future of laparoscopic renal surgery. Investig. Clin. Urol. 57 (Suppl 2):110-113.

Dominguez-Escrig JL, Vasdev N, O'Riordon A and Soomro N (2011). Laparoscopic partial nephrectomy: Technical considerations and an update. J. Minim. Access Surg. 7(4): 205-221.

Faiena I, Han CS and Olweny EO (2014). Laparoscopic partial nephrectomy: state of the art review. Open Acc. Surg. 7: 59-69.

Fariborz B, Pusztai C, Farkas L, Buzogány I, et al. (2017). Laparoscopic partial nephrectomy in warm ischemia: impact of parenchymal loss on kidney function. Magyar Urol. 2017(4): 158-166.

Guillonneau B, Gill IS, Janetschek G and Tuerk IA (2009). Laparoscopic Techniques in Uro-Oncology. London: Springer-Verlag, pp. 71-83.

Jimenez-Romero ME, Moreno-Cortes JC, Canelon-Castillo EY, Diez-Farto S, et al. (2019). Predictive Factors of Renal Function in Partial Laparoscopic Nephrectomy in Patients with a Kidney Tumor. Curr. Urol. 13(3): 150-156.

Kapoor A (2009). Laparoscopic partial nephrectomy: A challenging operation with a steep learning curve. Can. Urol. Assoc. J. 3: 119-120.

Kutikov A and Uzzo RG (2009). The R.E.N.A.L. nephrometry score: a comprehensive standardized system for quantitating renal tumor size, location, and depth. J. Urol. 182: 844-853.

Luk ACO, Pandian RMK and Heer R (2018). Laparoscopic renal surgery is here to stay. Arab J. Urol. 16(3): 314-320.

Nadu AM, Laufer M, Winkler H, Kleinmann N, et al. (2007). Laparoscopic partial nephrectomy: Single center experience with 140 patients-evolution of the surgical technique and its impact on patient outcomes. J. Urol. 178: 435-439.

Rendon RA, Kapoor A, Breau R, Leveridge M, et al. (2014). Surgical management of renal cell carcinoma: Canadian Kidney Cancer Forum Consensus. Can. Urol. Assoc. J. 8(5-6): e398-e412.

Shikanov S, Lifshitz D, Chan AA, Okhunov Z, et al. (2010). Impact of ischemia on renal function after laparoscopic partial nephrectomy: A multicenter study. J. Urol. 183: 1714-1718.

Trần NS, Châu QT, Dương QV, Trần TT, et al. (2010). Phẫu thuật nội soi sau phúc mạc cắt bán phần thận trong bướu thận: Giải pháp hài hòa trong ung thư học. Y Học TP HCM. 14(1): 27-31.

van Oostenbrugge TJ, Fütterer JJ and Mulders PF (2018). Diagnostic imaging for solid renal tumors: a pictorial review. Kidn. Canc. 2(2): 79-93.

Vũ NKCV (2015). Nhận xét đặc điểm lâm sàng, cận lâm sàng và kết quả điều trị phẫu thuật ung thư thận tại bệnh viện Việt Đức từ năm 2012 đến 2014. Y học TP HCM. 19(4): 264-269. 
Wolf JS (2010). Laparoscopic partial nephrectomy. Current clinical urology: Essential urologic laparoscopy. Humana Press, pp.165-184.

Yang F, Zhou Q, Li X and Xing N (2019). The methods and techniques of identifying renal pedicle vessels during retroperitoneal laparoscopic radical and partial nephrectomy. W. J. Surg. Oncol. 17(1): 1-8. 